\title{
Changes in RANKL during the first two years after cART initiation in HIV-infected CART naïve adults
}

Inger Hee Mathiesen ${ }^{1}$, Mohammad Salem², Jan Gerstoft ${ }^{1}$, Julie Christine Gaardbo ${ }^{1}$, Niels Obel ${ }^{1}$, Court Pedersen ${ }^{3}$, Henrik Ullum ${ }^{4}$, Susanne Dam Nielsen ${ }^{1}$ and Ann-Brit Eg Hansen ${ }^{5 *}$

\begin{abstract}
Background: By assessing the changes in concentration of soluble receptor activator of nuclear factor $\mathrm{k} B$ ligand (RANKL) and osteoprotegrin (OPG) after initiation of combination antiretroviral therapy (cART) in treatment-naïve HIVinfected patients we aimed to evaluate whether the initial accelerated bone loss could be mediated by increased soluble RANKL (sRANKL) levels associated with CD4+ T cell recovery.
\end{abstract}

Methods: We used multiplex immunoassays to determine sRANKL and OPG concentrations in plasma from 48 HIV patients at baseline and 12, 24, 48 and 96 weeks after CART initiation.

Results: Soluble RANKL changed significantly over time (overall $p=0.02$ ) with $25 \%$ decrease ( $95 \% \mathrm{Cl}:-42$ to -5 ) at week 24 compared to baseline and stabilized at a lower level thereafter. We found no correlation between CD4+ T cell count increment and changes in SRANKL or between percentage change in BMD and changes in SRANKL.

Conclusion: In this study there was no indication that the accelerated bone loss after CART initiation was mediated by early changes in sRANKL due to CD4+ T cell recovery. Future studies should focus on the initial weeks after initiation of CART.

Trial registration: Clinical-Trial.gov. id NCT00135460, August 25, 2005. The study was approved by the Danish Data Protection Agency, Danish Medicines Agency and Regional Ethics Committee.

Keywords: RANKL, OPG, CART, BMD, HIV infection

\section{Background}

HIV-infected patients experience a loss in bone mineral density (BMD) during the first 6-12 months after initiation of combination antiretroviral therapy (cART) [1-5]. The bone loss associated with cART initiation has been observed for all combinations of drug classes [6] although Tenofovir disoproxil fumarate (TDF) containing regimens are associated with more pronounced decreases in BMD of an additional $1.4 \%-2.0 \%[3,7]$. The molecular mechanisms behind the accelerated bone loss are not fully understood.

A key system in bone turnover is the RANK/RANKL/ OPG system [8]. Receptor activator of Nuclear Factor $\mathrm{K}$

\footnotetext{
* Correspondence: ann-brit.eg.hansen.02@regionh.dk

${ }^{5}$ Department of Infectious Diseases, Copenhagen University Hospital Hvidovre Hospital, Kettegaard Allé 30, -2650 Copenhagen, DK, Denmark Full list of author information is available at the end of the article
}

$B$ (RANK) expressed on osteoclast surfaces binds receptor activator of nuclear factor $\mathrm{k} B$ ligand(RANKL), which is expressed on osteoblast surfaces but also secreted by endothelial cells, B lymphocytes and T lymphocytes [9]. Binding of RANKL to RANK initiates a cascade of intracellular pathways which activates gene transcription necessary for osteoclast maturation and activity [9]. Osteoprotegrin (OPG) is produced by osteoblasts and works as a decoy receptor of RANKL thus inhibiting osteoclast activity [9].

A study of HIV-1 transgenic rats reported increased bone degradation and a shift in the ratio of RANKL to OPG favouring osteoclastogenesis and hence bone loss [10], findings which were later validated in HIV positive subjects [11]. A further study in T-cell depleted knockout mice demonstrated that transfusion of T-cells corresponded to increased RANKL concentrations and bone 
loss (BMD evaluated by DXA scan) within the repleted mice [12]. In other inflammatory conditions such as chronic colitis, activated CD4 + T cells have been shown to produce RANKL [13]. These data suggest that the immune reconstitution and/or CD4 $+\mathrm{T}$ cell recovery after cART initiation could be a contributing factor to bone loss. In a previous study [1], we have shown a significant bone loss after cART initiation at the spine and femoral neck after 24 and 48 weeks, respectively. Furthermore, more pronounced bone loss was found in individuals with very low CD4 + T cell count at time of cART initiation. Other prospective studies have also found that low baseline CD4 $+\mathrm{T}$ cell count [3], especially CD4 $+\mathrm{T}$ cell count below 50 cells $/ \mathrm{mm}^{3}[14,15]$, was a predictor of more pronounced bone loss after cART initiation.

In the present study, we aimed to assess the changes in soluble RANKL (sRANKL) and OPG repeatedly over 96 weeks in treatment-naïve HIV-infected patients initiating cART. We hypothesized that treatment with cART would result in increased sRANKL concentration and altered RANKL/OPG ratio during the first months after cART initiation due to CD4 $+\mathrm{T}$ cell recovery. We also hypothesized that the early increase in sRANKL concentrations would be negatively correlated with baseline CD4 $+\mathrm{T}$ cell count. Finally, we hypothesized that sRANKL and RANKL/OPG ratio would correlate negatively with BMD.

\section{Methods}

\section{Study population}

The SPAR BMD study has been described previously [1]. In this study patients from three Danish centers specialized in HIV treatment were randomized to a protease inhibitor (PI) sparing regimen (zidovudine/lamivudine/ efavirenz) or a nucleoside reverse transcriptase inhibitor (NRTI) sparing regimen (lopinavir/ritonavir/efavirenz). Site specific Dual X Ray Absorptiometry (DXA) scans were performed to evaluate spine and femoral neck BMD at baseline and week 24, 48, 96 and 144 after initiation of cART. In the present study, we used stored plasma samples from patients from the two largest centers to measure sRANKL and OPG at baseline and week 12, 24, 48 and 96. Three patients received oral corticosteroids during the study period; two patients within the first study year, and one patient within the second study year. No patients received bisphosphonates or other anti-osteoporotic medication during the study period.

\section{Measurement of soluble RANKL and OPG}

At baseline, week 12, 24, 48 and 96 a 10 ml EDTA blood sample (non-fasting) was drawn. Samples were immediately centrifuged for $10 \mathrm{~min}$ at $3000 \mathrm{~g}$ at $0^{\circ}-4^{\circ}$ Celsius, then plasma was transferred to $\mathrm{Nunc}^{\mathrm{Tm}}$ tubes and frozen immediately at $-80^{\circ}$ Celsius until analysis.
Concentration of sRANKL and OPG were determined by multiplex analysis (Biomedica, Vienna, Austria) according to the manufacturer's instructions. For RANKL, $100 \mu \mathrm{l}$ of pre-prepared standards/controls and samples were added to the reaction wells of the microarray, and $100 \mu$ biotinylated anti soluble RANKL antibody was then added to each well. The plate was incubated over night at room temperature with continuous shaking. The following day the antibody-coupled microspheres were washed 5 times in pre-prepared wash buffer and incubated with $200 \mu \mathrm{l}$ streptavidin-alkaline phosphate for $1 \mathrm{~h}$ at room temperature. Then the plate was washed as above and $100 \mu \mathrm{l}$ of each amplifier A (inorganic salts and buffered enzyme solution with tetrazolium violet) and amplifier B (stabilized NADPH solution) per well were added and again incubated at room temperature for $45 \mathrm{~min}$. Finally, $50 \mu \mathrm{l}$ stop solution was added to each well and the absorbance was measured immediately at $490 \mathrm{~nm}$ with reference $630 \mathrm{~nm}$.

For OPG, $100 \mu \mathrm{l}$ assay buffer, $50 \mu \mathrm{l}$ standards/controls/samples, and $50 \mu \mathrm{l}$ biotinylated anti OPG antibody was mixed, added to plate, and incubated over night at room temperature with continuous shaking. After incubation, antibody-coupled microspheres were washed 5 times in wash buffer and incubated with $200 \mu$ l assay substrate for $20 \mathrm{~min}$ at room temperature. The reaction was then stopped as before and the absorbance was measured immediately at $450 \mathrm{~nm}$ with reference $630 \mathrm{~nm}$. According to the manufacturer the inter-assay $\mathrm{CV}$ was $<15 \%$ and intra-assay $\mathrm{CV}$ was $<10 \%$ for both RANKL and OPG. All reads were performed using a Luminex 100 System (Luminex 100 ${ }^{\mathrm{TM}}$ platform (Luminex Corp., Austin, TX, USA) and samples from the same participant were run on the same plate.

\section{Statistical analyses}

Baseline characteristics and measurements of sRANKL, OPG concentrations, CD4 + T cells and BMD and T scores were expressed as medians and interquartile range (IQR) or mean and standard deviation (SD) as appropriate. Differences between groups were compared using Mann Whitney $U$ test and $X^{2}$ test as appropriate. OPG, sRANKL and OPG/RANKL ratio were log2 transformed, and CD4 $+\mathrm{T}$ cell count was square rooted to obtain normal distribution. A linear mixed model (LMM) was applied to analyze mean changes in sRANKL and OPG concentrations over time and to analyze whether treatment group was associated with changes in sRANKL or OPG over time. LMM data was backtransformed and calculated as percent change from baseline. We used Student's $\mathrm{T}$ test to compare changes in sRANKL from baseline $(\triangle \mathrm{RANKL})$ at week 12 and 24 in patients with baseline CD4 $+\mathrm{T}$ cell count $<200$ cells $/ \mu$ l versus patients with baseline CD4 + T cell count $\geq 200$ cells/ $\mu$ l. Pearson's 
correlation was used to evaluate correlations between $\mathrm{CD} 4+\mathrm{T}$ cell increment $(\triangle \mathrm{CD} 4)$ and $\triangle \mathrm{RANKL}$ difference from baseline to week 12. Associations between baseline RANKL/OPG ratio and BMD and percentage change in BMD and $\triangle$ RANKL at week 12 were also analyzed. Multivariate linear regression was used to adjust for age, sex and BMI at baseline. Statistical analysis was performed with IBM SPSS Statistics 22. A $p$ value $\leq 0.05$ was considered significant.

\section{Results}

A total of 48 patients with baseline BMD and RANKL/ OPG measurements and at least one follow-up BMD and RANKL/OPG measurement participated in the BMD RANKL sub study (23 in the NRTI sparing and 25 in the PI sparing group). There were no differences in baseline characteristics (Table 1) and therefore groups were pooled in the following analyses.

Evolution of BMD over 144 weeks in the randomized trial has been described in details previously [1]. Major findings were a marked decrease in BMD at the spine and femoral neck at week 24 and 48 . In the present substudy, the percentage change from baseline at the lumbar spine was $-2.86(95 \% \mathrm{CI} ;-3.73$ to -1.99$)$ at week 24 , and -2.26 (95\% CI; -3.39 to -1.14$)$ at week 48 . The percentage change from baseline at the femoral neck was -2.59 $(95 \% \mathrm{CI} ;-4.61$ to -1.57$)$ at week 24 and -5.01 (95\%
CI; -6.42 to -3.58$)$ at week 48 . All changes were statistically significant $(p<0.001)$. There were no significant differences between the PI-sparing arm and the NRTI-sparing arm.

\section{Evolution of RANKL and OPG during CART treatment}

Over time, sRANKL changed significantly (overall $p=0.02$ ) with a decline and stabilization from week 24 and onwards.

The mean estimated percent change in sRANKL from baseline was $2 \%$ at week 12 (95\% CI: -20 to 31$),-25 \%$ ( $95 \%$ CI: -42 to -5 ) at week $24,-20 \%$ (95\% CI: -37 to 4 ) at week 48 and $-27 \%$ (95\% CI: -45 to -5 ) at week 96.

OPG levels did not change significantly over time (overall $p=0.32$ ) although there was a trend towards an increase from baseline to week 12 . The mean estimated change from baseline was $12 \%$ (95\% CI: 1-24) at week $12,5 \%(95 \% \mathrm{CI}:-6$ to 16$)$ at week $24,8 \%$ (95\% CI: -3 to 21 ) at week 48 , and $8 \%$ (95\% CI: -3 to 22) at week 96. Moreover, RANKL/OPG ratio decreased over time, but the changes were not statistically significant. There was no association between treatment group and changes in sRANKL over time (data not shown).

The mean levels of sRANKL, OPG and RANKL/OPG ratio are depicted in Table 2 and Fig. 1.

Table 1 Baseline characteristics

\begin{tabular}{|c|c|c|c|c|}
\hline Entry & Overall & NRTI sparing & PI sparing & $P$ level \\
\hline No & 48 & 23 & 25 & - \\
\hline Male, (\%) & $44(92)$ & $21(91)$ & $24(96)$ & 0.90 \\
\hline Age (years) & $41.9(38.12-53.72)$ & $41.30(38.2-50.9)$ & $41.90(38.1-54.6)$ & 0.67 \\
\hline Weight (kg) & $70.95(65.17-80.86)$ & $74(66-88.5)$ & $69.75(64.4-73.5)$ & 0.06 \\
\hline Height (cm) & $178(173.5-184.75)$ & $178(172.5-182)$ & $178(174-186)$ & 0.71 \\
\hline BMI $\left(\mathrm{kg} / \mathrm{cm}^{2}\right)$ & $22.03(20.48-24.94)$ & $23.92(21.3-25.76)$ & $21.65(20.46-22.84)$ & 0.06 \\
\hline CD4 + T cell count baseline (cells/ $\mu l)$ & $200(86-280)$ & $240(110-315)$ & $170(70-280)$ & 0.20 \\
\hline CD4 + T cell count nadir (cells/ $\mu l)$ & $160(86-250)$ & $200(110-250)$ & $140(68-240)$ & 0.21 \\
\hline Spine BMD $\left(\mathrm{g} / \mathrm{cm}^{2}\right)$ & $1.09(0.926-1.20)$ & $1.11(0.96-1.22)$ & $1.04(0.96-1.18)$ & 0.38 \\
\hline Spine T-score & $-0.34(-1.70-0.71)$ & $-0.15(-1.36-0.83)$ & $-0.71(-1.78-0.40)$ & 0.35 \\
\hline Spine Z-score & $-0.01(-1.34-0.85)$ & $0.00(-0.64-1.2)$ & $-0.12(-1.3-0.74)$ & 0.41 \\
\hline Hip BMD $\left(\mathrm{g} / \mathrm{cm}^{2}\right)$ & $0.89(0.78-0.96)$ & $0.90(0.81-0.95)$ & $0.86(0.78-0.96)$ & 0.70 \\
\hline Hip T-score & $-0.89(-1.67--0.34)$ & $-0.86(-1.5--0.37)$ & $-0.96(-1.63--0.44)$ & 0.78 \\
\hline Hip Z-score & $-0.34(-0.86-0.42)$ & $-0.31(-0.62-0.32)$ & $-0.37(-0.97-0.31)$ & 0.63 \\
\hline HIV RNA (log10 copies/ml) & $5.24(4.69-5.47)$ & $5.01(4.5-5.7)$ & $5.30(5.1-5.5)$ & 0.37 \\
\hline RANKL (pg/ml) & $7.79(2.83-12.01)$ & $8.72(2.79-12.93)$ & $7.38(2.8-11-93)$ & 0.83 \\
\hline $\mathrm{OPG}(\mathrm{pg} / \mathrm{ml})$ & 34.19 (26.53-45.75) & 37.73 (27.95-45.92) & 32.08 (24.19-51.72) & 0.93 \\
\hline RANKL/OPG ratio & $0.21(0.08-0.36)$ & $0.18(0.1-0.38)$ & $0.25(0.07-0.36)$ & 0.82 \\
\hline
\end{tabular}

Continuous data expressed as median and interquartile range (IQR), nominal data expressed as frequency (\%) $\mu$ l microliter, $k g$ kilograms, $c m$ centimeters, $g$ grams, $p g$ picograms 
Table 2 RANKL, OPG and CD4+ T cell count in overall cohort

\begin{tabular}{lllll}
\hline WEEK & SRANKL & SOPG & RANKL/OPG ratio & CD4 + T cells \\
\hline week 0 & $7.79(2.83-12.01)(n=48)$ & $34.19(26.53-45.75)(n=48)$ & $0.21(0.08-0.36)$ & $200(86-280)(n=48)$ \\
week 12 & $8.03(3.72-11.01)(n=45)$ & $40.52(28.55-53.67)(n=45)$ & $0.18(0.11-0.30)$ & $300(202-400)(n=48)$ \\
week 24 & $5.75(2.88-9.41)(n=45)$ & $35.83(25.54-50.66)(n=45)$ & $0.17(0.07-0.29)$ & $340(240-470)(n=47)$ \\
week 48 & $5.92(3.07-11.98)(n=43)$ & $36.10(26.19-55.82)(n=42)$ & $0.16(0.09-0.35)$ & $360(250-535)(n=45)$ \\
week 96 & $6.03(1.02-11.31)(n=34)$ & $38.24(29.82-50.47)(n=33)$ & $0.15(0.05-0.30)$ & $450(280-600)(n=43)$
\end{tabular}

Values are depicted as median and interquartile range (IQR). sRANKL and OPG in picograms/ml (pg/ml) and CD4+ T cells as cells/pl

\section{CD4+ T cell count and sRANKL concentrations}

CD4 $+\mathrm{T}$ cell counts are depicted in Table 2. $\triangle \mathrm{RANKL}$ at week 12 and week 24 did not differ significantly in the group of patients with CD $4+\mathrm{T}$ cells below 200 cells $/ \mu \mathrm{l}$ $(n=25)$ versus patients with CD4 + T cells above 200 cells/ $\mu \mathrm{l}(n=23)$ at baseline in crude analysis and when adjusted for body mass index(BMI), gender, and age (data not shown). Neither was there any correlation between CD4 + T cell increment and $\triangle \mathrm{RANKL}$ at week $12(r=0.039, p=0.81)$.

RANKL/OPG ratio, sRANKL increment and association with BMD at baseline and week 24

Baseline RANKL/OPG ratio was not significantly correlated with baseline spine or femoral neck BMD, $(r=0.04$ $p=0.78$ and $r=0.13 p=0.41$, respectively). This
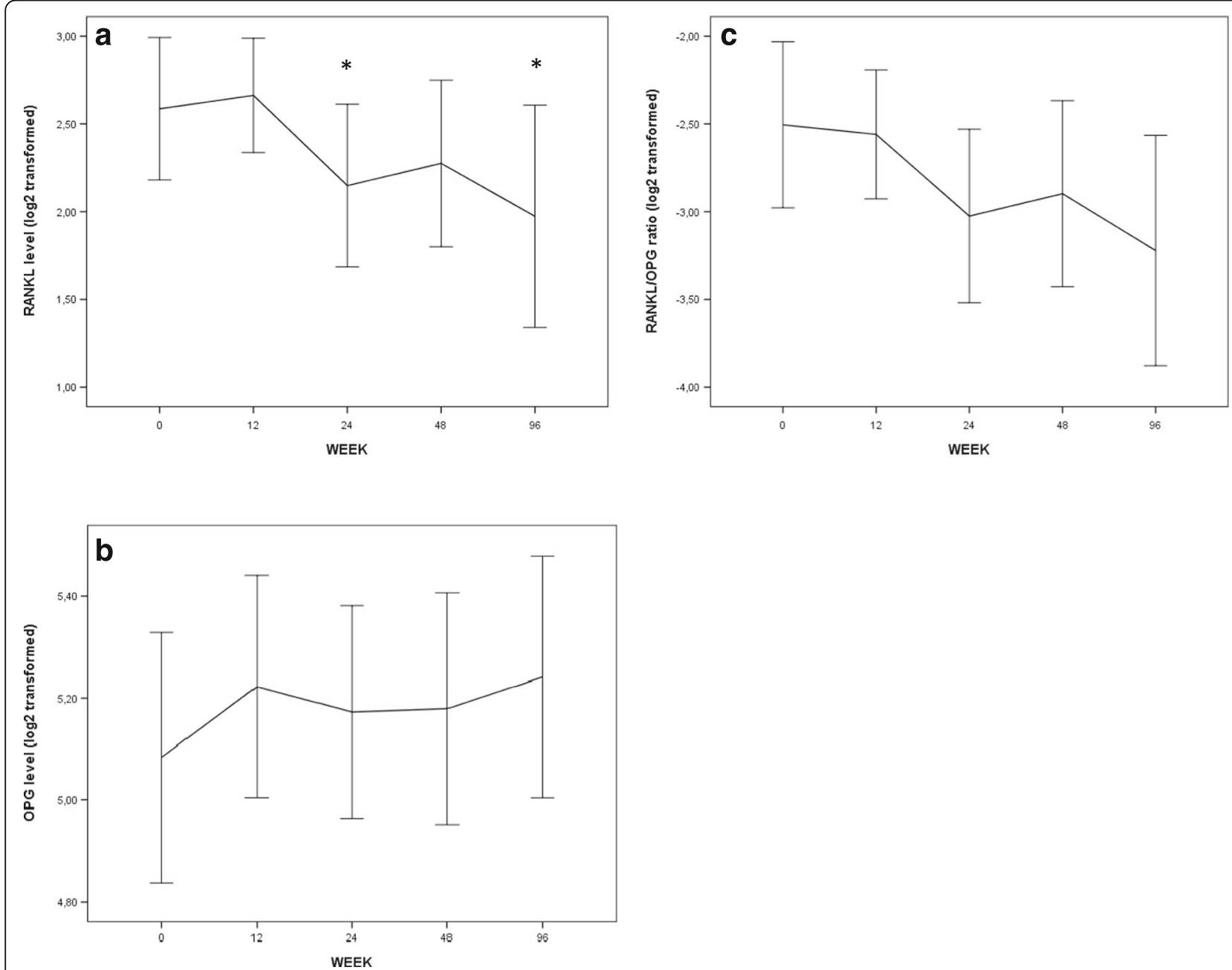

Fig. 1 Overall mean RANKL, OPG and RANKL/OPG ratio. Mean levels ( $95 \% \mathrm{Cl}$ ) of (1a) RANKL, (1b) OPG and (1c) RANKL/OPG ratio. Data are logarithmically transformed. 1 unit change in log2 unit corresponds to a doubling in original unit. ${ }^{*} p<0.05$ compared to baseline value 
remained insignificant after adjusting for BMI, gender and age in multivariate analysis (data not shown). There was no correlation between week 12 sRANKL increment and percentage changes in BMD at week 24 at the lumbar spine $(r=0.007 p=0.96)$ or at the femoral neck ( $r=0.059 p=0.71$ ), also not in analyses adjusted for age, BMI and gender.

\section{Discussion}

In the present study, we measured sRANKL and OPG at multiple time points within the first two years after cART initiation in treatment-naïve HIV-infected patients. sRANKL values had decreased at week 24 and stabilized at a lower level thereafter. The decrease might help to explain the stagnation of cART related bone loss after 6-9 months of therapy, as a decline in RANKL levels leads to decreased osteoclast activation. Similar results with a decline in RANKL levels 6 to 12 months after initiation of cART have been reported in other studies $[16,17]$.

In contrast to our hypothesis, we did not detect a significant increase in SRANKL at week 12 and our results do not explain the accelerated BMD loss occurring after cART initiation. Possibly, changes in soluble RANKL important for the accelerated bone loss may have taken place already before week 12, since it is well established that bone turnover, measured as increasing levels of C-terminaltelopeptide-cross-links (CTx, a marker of bone degradation and thereby osteoclast activity), and osteocalcin (OC, a protein secreted by mineralizing osteoblasts), is accelerated as early as two weeks after commencing cART $[16,18]$. The observed non-significant decrease in SRANKL to OPG ratio could reflect that we captured the resetting of bone metabolism after a catabolic window within the first weeks after cART initiation. However, recently published data [18] did not display any increase in plasma RANKL at week 2, where a marked increase in bone resorption markers had already taken place, indicating that sRANKL may not be the main mediator of bone loss. This is further underscored by the fact that BMD loss at week 24 did not correlate with sRANKL increment at week 12 in our study. Combining these results it seems, that the mechanism of cART induced bone loss was not linked directly to changes in sRANKL.

Indeed, it has been suggested, that the normal regulation of bone resorption and bone formation mediated by RANK/RANKL/OPG, is uncoupled in HIV treatmentnaïve patients in favour of increased osteoclast activity [16]. This will, when bone turnover is increased with cART initiation, lead to the observed accelerated bone loss. Another explanation of the lack of association between sRANKL and BMD loss could be, that sRANKL may not reflect what takes place in the bone microenvironment i.e. direct cross talk between membrane bound RANKL in osteoblasts and membrane bound RANK in osteoclasts, which may be of greater importance of osteoclast activation than soluble RANKL [19]. Unfortunately we did not have any bone turnover markers in order to evaluate bone turnover in context of the observed BMD reductions.

Despite animal models having shown a positive correlation between increasing CD4 $+\mathrm{T}$ cell count and sRANKL and BMD loss [12], we did not find any association between changes in sRANKL and baseline CD4 $+\mathrm{T}$ cell count or magnitude of CD $4+\mathrm{T}$ cell recovery in our present study. In contrast, a recently published study observed significantly increased RANKL levels 12 and 24 weeks after cART initiation coinciding with CD4 + T cell recovery in a group of patients initiating cART ( $n=19,18]$. These diverting results may be attributable to several differences in study design including study population size ( $n=48$ vs $n=19)$, difference in ethnic origin and a TDF-containing regimen in the study by Ofotokun et al. We also had a lower proportion of patients with very low CD4 cell counts. In our study, only five patients $(10 \%)$ had baseline CD4 + T cell count below 50 cells/ $\mu \mathrm{l}$, which may have hampered the power to find an association between CD4+ T cell reconstitution, changes in sRANKL and changes in BMD.

The major strength of this prospective study is that we repeatedly measured sRANKL within the two first years after cART initiation including measurements at week 12 and 24, the period where the accelerated bone loss induced by cART initiation peaks. Only one other group has successfully measured sRANKL levels at multiple time points within 24 weeks of cART initiation [18]. In our study, the patients were randomized to a cART regimen with or without NRTIs. Although the regimens are less modern regimens, the design ensured that we were evaluating the immunological effect of treatment initiation rather than the direct drug effect of NRTIs or TDF.

Our study has several limitations. The study population is relatively small and there is a risk of type II error given the large biological variability of sRANKL [18]. We did not measure any proresorptive cytokines such as Tumor Necrosis Factor-alpha, interleukin-1 or interleukin-6 or any bone turnover markers which would help characterizing the role of sRANKL on bone turnover just after cART initiation. The degree of cross validation between different RANKL immune and ELISA assays are not well established making it difficult to directly extrapolate results between studies [20]. Further, blood sampling in this study was not performed fasting, which may affect within subject variability; and we only measured sRANKL and not RANKL expression by B-cells, other immune cells or osteoblasts. Finally, the majority of participants were males, and findings may not apply to women. 


\section{Conclusion}

In summary, we found that sRANKL decreased to levels below baseline values from week 24 and onwards after initiation of cART. In our study, in which median baseline CD4 + T cell count was around 200 cells/ $\mu$ l, there was no indication that the early cART induced accelerated bone loss was mediated through increased levels of sRANKL induced by CD4+ $\mathrm{T}$ cell recovery. Further studies focusing on the initial weeks after initiation of cART are warranted.

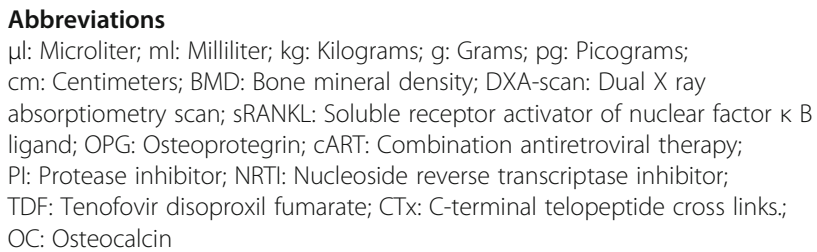

\section{Acknowledgements}

We gratefully acknowledge the participants who made this study possible.

\section{Funding}

Not applicable.

\section{Availability of data and materials}

The dataset used and analyzed during the current study is available from the corresponding author on request.

\section{Authors' contributions}

$\mathrm{ABH}, \mathrm{SD}, \mathrm{HU}, \mathrm{NO}, \mathrm{JG}$ and $\mathrm{CP}$ participated in design of the study and provided clinical data. ABH, JCG and MS made substantial contributions to data acquisition and provided clinical data. MS and HU performed immunoassays. IHM conducted statistical analyses and interpreted data. IHM compiled the first draft of the study manuscript and all authors contributed to subsequent revisions. All authors read and approved the final manuscript.

\section{Competing interests}

CP has received research grant from Gilead, and has received salary for teaching from Abbott and MSD.

JG has received honoraria for consulting and presenting paid to his institution from Gilead, Abbvie, ViiV, BMS, MSD, Janssen, and Medivir.

SDN has received unrestricted research grants from Novo Nordisk Foundation, Lundbeck Foundation, Rigshospitalet Research Council. Travelling grants from Gilead, MSD, BMS and GSKViiV. Advisory board activity for Gilead and GSKViiV. $\mathrm{IHM}, \mathrm{ABH}, \mathrm{MS}, \mathrm{JCG}, \mathrm{HU}, \mathrm{NO}$ all declare no competing interests.

\section{Consent for publication}

\section{Not applicable.}

\section{Ethics approval and consent to participate}

Clinical-Trial.gov. id NCT00135460, first registered August 25, 2005. Further, the study was approved by the Danish Data Protection Agency, Danish Medicines Agency and Regional Ethics Committee.

Patients provided signed informed consent before participating according to good clinical practice standards.

\section{Publisher's Note}

Springer Nature remains neutral with regard to jurisdictional claims in published maps and institutional affiliations.

\section{Author details}

'Department of Infectious Diseases, Copenhagen University Hospital Rigshospitalet, Copenhagen, Denmark. ${ }^{2}$ Department of Microbiology \& Immunology, Hollings Cancer Center, Medical University of South Carolina, Charleston, SC, USA. ${ }^{3}$ Department of Infectious Diseases, Odense University Hospital, Odense, Denmark. ${ }^{4}$ Department of Clinical Immunology,
Copenhagen University Hospital Rigshospitalet, Copenhagen, Denmark. ${ }^{5}$ Department of Infectious Diseases, Copenhagen University Hospital Hvidovre Hospital, Kettegaard Allé 30, -2650 Copenhagen, DK, Denmark.

Received: 4 November 2016 Accepted: 30 March 2017

Published online: 11 April 2017

\section{Reference}

1. Hansen AB, Obel N, Nielsen H, Pedersen C, Gerstoft J. Bone mineral density changes in protease inhibitor-sparing vs. nucleoside reverse transcriptase inhibitor-sparing highly active antiretroviral therapy: data from a randomized trial. HIV Med. 2011;12:157-65.

2. Brown TT, Moser C, Currier JS, Ribaudo HJ, Rothenberg J, Kelesidis T, et al. Changes in Bone Mineral Density After Initiation of Antiretroviral Treatment With Tenofovir Disoproxil Fumarate/Emtricitabine Plus Atazanavir/Ritonavir, Darunavir/Ritonavir, or Raltegravir. J Infect Dis. 2015;212:1241-9.

3. McComsey GA, Kitch D, Daar ES, Tierney C, Jahed NC, Tebas P, et al. Bone mineral density and fractures in antiretroviral-naive persons randomized to receive abacavir-lamivudine or tenofovir disoproxil fumarate-emtricitabine along with efavirenz or atazanavir-ritonavir: Aids Clinical Trials Group A5224s, a substudy of ACTG A5202. J Infect Dis. 2011;203:1791-801.

4. Stellbrink HJ, Orkin C, Arribas JR, Compston J, Gerstoft J, Van WE, et al. Comparison of changes in bone density and turnover with abacavirlamivudine versus tenofovir-emtricitabine in HIV-infected adults: 48-week results from the ASSERT study. Clin Infect Dis. 2010;51:963-72.

5. van Vonderen MG, Lips $P$, van Agtmael MA, Hassink EA, Brinkman K, Geerlings SE, et al. First line zidovudine/lamivudine/lopinavir/ritonavir leads to greater bone loss compared to nevirapine/lopinavir/ritonavir. AIDS. 2009: 23:1367-76.

6. Brown TT, McComsey GA, King MS, Qaqish RB, Bernstein BM, da Silva BA. Loss of bone mineral density after antiretroviral therapy initiation, independent of antiretroviral regimen. J Acquir Immune Defic Syndr. 2009; 51:554-61.

7. Yin MT, Overton ET. Increasing clarity on bone loss associated with antiretroviral initiation. J Infect Dis. 2011;203:1705-7.

8. Khosla S. Minireview: the OPG/RANKL/RANK system. Endocrinology. 2001; 142:5050-5.

9. Zaidi M. Skeletal remodeling in health and disease. Nat Med. 2007;13:791-801.

10. Vikulina T, Fan X, Yamaguchi M, Roser-Page S, Zayzafoon M, Guidot DM, et al. Alterations in the immuno-skeletal interface drive bone destruction in HIV-1 transgenic rats. Proc Natl Acad Sci U S A. 2010;107:13848-53.

11. Titanji K, Vunnava A, Sheth AN, Delille C, Lennox JL, Sanford SE, et al. Dysregulated B cell expression of RANKL and OPG correlates with loss of bone mineral density in HIV infection. PLoS Pathog. 2014;10:e1004497.

12. Ofotokun I, Titanji K, Vikulina T, Roser-Page S, Yamaguchi M, Zayzafoon M, et al. Role of T-cell reconstitution in HIV-1 antiretroviral therapy-induced bone loss. Nat Commun. 2015;6:8282

13. Totsuka T, Kanai T, Nemoto Y, Tomita T, Okamoto R, Tsuchiya K, et al. RANKRANKL signaling pathway is critically involved in the function of CD4+CD25 + regulatory T cells in chronic colitis. J Immunol. 2009:182:6079-87.

14. Moyle GJ, Hardy H, Farajallah A, McGrath SJ, Kaplita S, Ward D. Changes in bone mineral density after 96 weeks of treatment with atazanavir/ritonavir or lopinavir/ritonavir plus tenofovir DF/emtricitabine in treatment-naive patients with HIV-1 infection: the CASTLE body composition substudy. J Acquir Immune Defic Syndr. 2015;68:40-5.

15. Grant PM, Kitch D, McComsey GA, Dube MP, Haubrich R, Huang J, et al. Low baseline CD4+ count is associated with greater bone mineral density loss after antiretroviral therapy initiation. Clin Infect Dis. 2013;57:1483-8.

16. Brown TT, Ross AC, Storer N, Labbato D, McComsey GA. Bone turnover, osteoprotegerin/RANKL and inflammation with antiretroviral initiation: tenofovir versus non-tenofovir regimens. Antivir Ther. 2011;16:1063-72.

17. Bernardino Jl, Mocroft A, Mallon PW, Wallet C, Gerstoft J, Russell C, et al. Bone mineral density and inflammatory and bone biomarkers after darunavir-ritonavir combined with either raltegravir or tenofovir-emtricitabine in antiretroviral-naive adults with HIV-1: a substudy of the NEAT001/ANRS143 randomised trial. Lancet HIV. 2015;2:e464-73.

18. Ofotokun I, Titanji K, Vunnava A, Roser-Page S, Vikulina T, Villinger $F$, et al. Antiretroviral therapy induces a rapid increase in bone resorption that is positively associated with the magnitude of immune reconstitution in HIV infection. AIDS. 2016;30:405-14. 
19. Hikita A, Yana I, Wakeyama H, Nakamura M, Kadono Y, Oshima Y, et al. Negative regulation of osteoclastogenesis by ectodomain shedding of receptor activator of NF-kappaB ligand. J Biol Chem. 2006;281:36846-55.

20. Bowsher RR, Sailstad JM. Insights in the application of research-grade diagnostic kits for biomarker assessments in support of clinical drug development: bioanalysis of circulating concentrations of soluble receptor activator of nuclear factor kappaB ligand. J Pharm Biomed Anal. 2008;48: $1282-9$.

Submit your next manuscript to BioMed Central and we will help you at every step:

- We accept pre-submission inquiries

- Our selector tool helps you to find the most relevant journal

- We provide round the clock customer support

- Convenient online submission

- Thorough peer review

- Inclusion in PubMed and all major indexing services

- Maximum visibility for your research

Submit your manuscript at www.biomedcentral.com/submit 